\title{
Einsatzmöglichkeiten der Mitarbeiterbefragung
}

\author{
Stephan Sandrock
}

Die Qualität eines Produktes, einer Dienstleistung, der betriebsinternen Prozesse, der Führung etc. sind Erfolgsfaktoren eines Unternehmens. Eine kontinuierliche Überprüfung dieser Aspekte ist erforderlich, um diese nachhaltig zu gewährleisten; diese kann unter anderem mithilfe einer Mitarbeiterbefragung erfolgen.

Ziel einer MAB ist die Analyse von Stärken und Schwächen des Unternehmens, die in moderierten Workshops mit Mitarbeitenden und Führungskräften weiter geklärt und zu konkreten Maßnahmen führen sollen.

Die MAB verfolgt unterschiedliche Ziele: Analyse der Unternehmenskultur, des Betriebsklimas oder des Führungsverhaltens und anderes mehr. Exemplarische Einsatzmöglichkeiten der Mitarbeiterbefragung sind:

- Erfassung eines allgemeinen Stimmungsbildes (Wertungen, Erwartungen, Anforderungen und Bedürfnisse der Beschäftigten)
- Meinungserhebung zu aktuellen unternehmensrelevanten Themen

- Erfassung der Akzeptanz sowie Einleitung geplanter beziehungsweise bevorstehender Veränderungen

- Kontrolle und Evaluation von Veränderungsprozessen infolge bereits erfolgter Maßnahmen

- Datenlieferant für die Balanced Scorecard

- Datenlieferant für das interne beziehungsweise externe Benchmarking - Analyse des Bedarfs an Personal- und Organisationsentwicklung

- Optimierung der Arbeits- und Geschäftsprozesse

- Optimierung der internen Kommunikation

- Erhebung der Anlässe für Fluktuation beziehungsweise Fehlzeiten

- Selbstbewertung im Rahmen des Qualitätsmanagements (DIN EN ISO 9000 ff.)

- Aufbau eines Total-Quality-Managements (TQM) zum Beispiel mit dem EFQM-Modell 